\title{
Applying Internet of Things in Healthcare: A survey
}

\section{ISSN: 2640-9666}

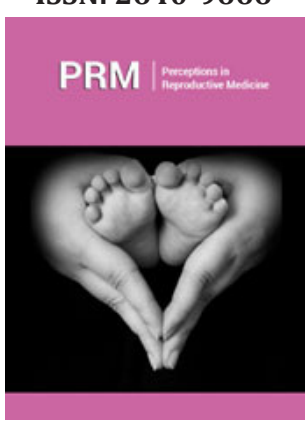

*Corresponding author: Ioannis-John Kosmas, Department of Telematics and Informatics, Harokopio University of Athens, Greece, Email: i.kosmas@hua.gr

Submission: 㘹 March 05, 2021

Published: 䟧 March 16, 2021

Volume 4 - Issue 4

How to cite this article: John Kosmas I, Papadopoulos T, Michalakelis C. Applying Internet of Things in Healthcare: A survey. Perceptions in Reproductive Medicine. 4(3). PRM. 000592. 2021.

DOI: $10.31031 /$ PRM.2021.04.000592

Copyright@ Ioannis-John Kosmas, This article is distributed under the terms of the Creative Commons Attribution 4.0 International License, which permits unrestricted use and redistribution provided that the original author and source are credited.

\author{
Ioannis-John Kosmas*, Papadopoulos T and Michalakelis C \\ Department of Telematics and Informatics, Harokopio University of Athens, Greece
}

\begin{abstract}
People nowadays are living in the era of advanced technology in their everyday live. In the context of the Internet of Things (IoT), the internet connectivity has been extended beyond standard devices, such as desktops, laptops, smartphones and tablets, to any range of traditionally dumb or non-internet-enabled physical devices and everyday objects. In recent years, IoT has taken over most of the industrial area, especially automation and control [1,2]. At the same time, IoT is applicable to other crucial sectors and especially health, since it offers numerous opportunities to improve health services and also enables health professionals (doctors, nurses, etc.) to make decisions based on real data. The IoT provides many additional health applications with the specific aim of monitoring patient progress and providing doctors with information on decision making. IoT health concerns and future prospects are presented in this paper based on a thorough survey of previous literature. Inspection of the results reveals that some health-related services are not yet sufficiently developed and interestingly enough the literature is limited in these services and there is no knowledge about the implementation of IoT in the health sector. This paper attempts to investigate the scientific gap in the IoT healthcare sector and it also gives interesting directions for future researches regarding the implementation of health data.
\end{abstract}

Keywords: IoT; Healthcare; Survey; Systematic literature review

\section{Introduction}

People nowadays are living in the era of advanced technology, where every part of their daily lives is related to some kind of technology in one way or another. A frequent phenomenon, which proves the above assumption, is the direct relationship of almost everyone with the mobile phones. People today have the opportunity to use the variety of software applications, via the Internet, and be informed about the weather forecasting, the banking and stock trading, the airline flights and other interesting aspects of their everyday life by either paying for these services through in-app transactions or not. Living in a connected world creates a grid of relationships but not only for smartphones and tablets. In the context of the Internet of Things (IoT), the internet connectivity has been extended beyond standard devices, such as desktops, laptops, smartphones and tablets, to any range of traditionally dumb or noninternet-enabled physical devices and everyday objects. Embedded with technology, these devices can communicate and interact over the internet, and they can be remotely monitored and controlled. In light of this, a great variety of additional smart devices of all sorts, equipped with sensors like home appliances, vehicles, energy consumption, temperature, medical devices, square measurements are dynamically connected to the net. These devices communicate with one another via Machine-to-Machine (M2M) technology. In the context of M2M the devices communicate and exchange information, either wired or wireless, through advanced next generation (NGAs) networks. In recent years, IoT has taken over most of the industrial area, especially automation and control. At the same time, IoT is applicable to other crucial sectors and especially health, since it offers numerous opportunities to improve health services and also enables health professionals (doctors, nurses, etc.) to make decisions based on real data. There is a powerful interest in wireless sensing element networks (the basic trigger mechanism for IoT primarily based systems), like the event of routing protocols and a spread of applications like medical imaging $[3,4]$.

The connections in the context of IoT health sector, refer to objects such as patient devices, physician patients, doctor-based devices, sensor-to-device devices. The IoT provides many additional health applications with the specific aim of monitoring patient progress and providing doctors with information on decision making in this respect [5-10]. Various medical 
devices, sensors, diagnostic and imaging devices seem to be good devices or objects and represent a significant a part of IoT. Internetbased healthcare services are expected to increase quality of life and enrich the user expertise. On the provider's perspective, IoT has the power to reduce time-consuming of the device by providing a distant management.

Taking into account previous literature, IoT health concerns and future prospects are presented in this paper. Thorough inspection of previous literature reveals that some health-related services are not yet sufficiently developed and interestingly enough the literature is limited in these services and there is no knowledge about the implementation of IoT in the health sector. This paper attempts to investigate the scientific gap in the IoT healthcare sector and it also gives interesting directions for future researches regarding the implementation of health data.

\section{What has been written for the IoT?}

The thought of net of things (Internet of Things or IOT) isn't new, the mix of computers and networks for observation and dominant the devices has been used for many years. One example of this, since the late Nineteen Seventies, was the telecommunication systems of electrical networks via phone lines wherever they were commercially used [11]. Today, the Internet of things is being exploited in many areas of everyday life, providing the convenience of technology to reach people's goals. Despite its wider expansion, there is no specific definition. The International Telecommunication Union (ITU) has attempted to do so. This association defines the web of Things as a worldwide info society infrastructure linking all varieties of things supported existing and practical info and communication technologies [12].

During the 1990s, the machine-to-machine or M2M solution was presented, as a result of advances in wireless technology and made operational and industrial solutions known to the public for monitoring and operating equipment. Several of these first M2M solutions, however, have found application in closed networks and were created for specific purposes on specific standards (mainly industrial), rather than on IP-based networks and on web standards [13]. It was an IP-enabled toaster, powered and disconnected via the Internet - for the first time as a "device" on the Internet - and presented at an internet conference in 1990 [14]. As a result of this presentation, other "things" were created which were associated with an IP, such as e.g. a drink machine at Carnegie Mellon University within the America and a kitchen appliance at Trojan area at the University of Cambridge within the uk [15]. At the beginning of the 2000s, a pioneering scientist, Kevin Ashton, laid the foundations for IoT in MIT's AutoID lab. Ashton introduced this new proposal looking for ways to enhance Proctor \& Gamble's activities by linking RFID (Radio Frequency Identification) info to the web. His idea was based on something very simple. If there have been instrumentality with identifiers and wireless property to things in daily life, these could be manageable via computers and communicate with each other.

Based on the above principles, the current Internet of Things has established a foundation and a strong field of research and development in "Networking Smart Objects". In 2002, the National Science Foundation (NSF) revealed a report on Convergence Technologies for the development of Human Performance, technology, info Technology and scientific discipline, NSF/DOCsponsored Report, 2002 that targeted on desegregation Info and Communication Technology (ICT) technology to dramatically improve the standard of lifetime of individuals and also the productivity of countries. the primary ITU report in 2005 projected to mix net technology with beholding technologies, wireless networks, sensors, a constitutional system and nanotechnologies to attach things to the planet so things are noticed, derived and controlled via the web. IoT consists of a collection of technologies that support the communication and interaction between a good vary of networked devices and devices. Internet-based business systems are developed for numerous applications like health care systems industrial setting.

In 2009, Kirizis projected a replacement definition for good product supported what happened to United States of America as a personality's being. It proposes lifecycle management of electric circuit merchandise to develop a lot of increased product knowledge technologies which will be utilized in the long run to develop intelligent or intelligent merchandise and conjointly address not solely static however conjointly dynamic product knowledge. In year 2009, have projected a platform for making associate degree intelligent application for wireless devices and sensors. He works performed in 2010 focuses on new security measures and varied IoT privacy challenges. It utilizes varied parameters like attacks, information authentication, access management and client privacy to review the privacy challenges and build a brand-new security model. This document conjointly describes the importance of putting in a task force to conduct analysis into the legal challenges of IoT. It conjointly proposes to make a legal framework that's additional versatile and straightforward to adapt to the particular wants.

In 2010, focuses on describing all the most important terminologies used on the net of Things, by elucidative the foremost vital terms like things, devices and entities of interest, resources, confrontation, identity and, most significantly, relationships between them. In 2010, planned wireless sensing element network technologies supported numerous customary protocols, net protocols to facilitate the net of Things. It focuses on the way to adapt the informatics to the realm of things. this text introduces sensing element networks for a worldwide reach (SNAIL) approach to IoT. It conjointly includes four major network protocols: quality, net activation, time synchronization, and security. The year 2013 urged to observe gas escape with the assistance of GSM mobile phones.

They used 2 gas sensors to observe gas leaks in a very specific location and 8051 microcontrollers, programmed within the programming language, and GSM phone. The GSM phone was organized to send gas escape notifications within the style of a brief Message Service (SMS) message, that indicates the precise location on another GSM phone to activate the immediate action required. All this method can result in quicker detection once gas 
escape happens. In 2013 propose to make Associate in Nursing IoT supported accidental, occasional networking of devices (eg mobile and sensible vehicles) exploitation short-range radio techniques (e.g. Bluetooth and Wi-Fi). this can produce an in-depth relationship between the human and opportunist connections of sensible things as a result of it deals with the promotion and dissemination of data among and between opportunist communities that square measure formed by movement and occasional contact with humans. This document proposes a \{way|a technique $\}$ of making the net in a very totally different way so it may be created whenever it's required with the assistance of radio frequencies. In year 2014, planned a gas escape detection system and alerted the subscriber through the alarm and standing indication, whereas mechanically disabling the gas offer valve at an equivalent time. It mechanically uses a commonly closed coil valve to shut the gas valve before requesting help through visual show and alarm. mechanically opens the valve for traditional operations as before long because the escape falls below the point.

In 2014, specialize in however 2 devices in every IOT ought to communicate showing intelligence as a result of the standard of the data that's collected depends on however good the devices ar. within the IoT context, completely different \} devices work with different network standards, so that they will cause several network challenges, and this cannot be resolved with ancient routing protocols. Thus, this document planned prime routing algorithms, which may facilitate win good D2D communication at IoT. within the year 2016 prompt that one will use the IoT to make a network of varied connected designers and good sensors in order that this network will keep in mind the past and also the arrange for the long run. They additionally argued that the employment of enormous analytical knowledge to realize the specified SCC. He prompts that one may use mobile TV and cloud computing to make SCC and prompt that SCC would facilitate improve property, property and practicableness.

The I.o.T. system is the innovative that is transforming the healthcare environment for the healthcare sector by rising operating expenses and supporting caregivers. Using this technology, integration to healthcare systems through internet cloud is feasible, which provides clinicians the opportunity to view knowledge in real time that helps them to make educated choices, as well as provide evidence-based treatment. This initiative mostly helps regulate power use by controlling electronic items including the lights and fans from a distant location while reducing energy wastage. Today's world, concentrating on healthcare education and also the development of wireless communication has become significant. To meet our needs, IOT made it easy to wirelessly monitor, manage stuff in real time, use the internet. Thanks to the usage of the wireless sensor network in a healthcare system, we will the wire network issues and shift a healthcare system from one place to another place.

\section{Description of the Method}

The quality analysis performed in this paper is based on the Systematic Literature Review (SLR) as a secondary style of study.
According to Kitchenham [6] the goal of the review might include the following choices:

1. Discover the main trend for a subject in a specific scientific field and potential comments for those subjects.

2. Analyze potential conflicts of theories, in methodology in order to judge and evaluate most of them.

3. Explore potential gaps in research and create new knowledge in a specific subject in order to propose themes for further investigation.

4. Light up problems or themes for better understanding.

In order to capture these scientific questions, we used SLR. Starting our research, a review protocol was designed based on the guidelines of SLR [7] in order to describe our steps. The main goal of this paper is to investigate potential gaps in existing studies and highlight new aspects that need to be further analyzed. The specific steps followed are presented below.

\section{Search process}

The automated research is limited between 2016 and 2018. The main reason for this is the increasing interest in research activities in the IoT during this time period. Our automated research selected only journal review papers during this particular period of time. The research took place during October and November 2018. The databases used are Science direct, PubMed and Google Scholar in order to find scientific surveys to better capture the answers for the aforementioned scientific questions.

The selected journal papers were reviewed and specific inclusion and exclusion criteria were set in order to investigate final insights of each research study. The keywords used for the present study are:

$$
\begin{aligned}
& \text { a. Healthcare internet of things } \\
& \text { b. Health } \\
& \text { c. IoT }
\end{aligned}
$$

\section{Inclusion and exclusion criteria}

The inclusion criteria for the reviewed articles are presented below

a) Published between 2016-2018

b) Review articles

c) Scientific Journals, conferences

d) The main subject of the review was about IoT in healthcare

e) Data Base science direct, pub med, google scholar, IEEE,

f) English language

g) Key words " healthcare internet of things" , "health", "IoT" ' abstracts or title

h) Open access 
The exclusion criteria for the reviewed articles follow:

1) Abstracts and titles that were not included in the keywords

2) Journal articles and conference papers that their content was no available (open access)

3) Publications were found in more of one database

4) Articles written in a different language of English

5) Article that have no deal with IoT

6) Master thesis or PhD thesis

7) Premiere studies

8) Articles no exist in database Science direct or Pub Med or google Scholar

After a thorough literature review, the numbers of scientific papers that met the selection criteria in each stage are presented in Table 1.

Table 1: Meeting the selection criteria for each phase.

\begin{tabular}{|l|c|c|c|}
\hline \multicolumn{1}{|c|}{ Phase } & Searching & Keywords & $\begin{array}{c}\text { Number of } \\
\text { Articles }\end{array}$ \\
\hline 1 & $\begin{array}{c}\text { Years of publication } \\
2016-2018\end{array}$ & $\begin{array}{c}\text { Healthcare Internet } \\
\text { of Things, Health, } \\
\text { IoT, }\end{array}$ & 4125 \\
\hline 2 & Literature Review & & 334 \\
\hline 3 & Open Access & & 42 \\
\hline 4 & Exclusion criteria & & 4 \\
\hline
\end{tabular}

\section{Quality assessment}

According to the Centre for Reviews and Dissemination (CRD) of the York University, and more specifically the based on the criteria of the Database of Abstracts of Reviews of Effects (DARE) [8] the factors are supported four questions:

a) Are the inclusion and exclusion criteria of the reviews described, appropriate?

b) Is the search of the literature likely to own covered all relevant studies?

c) Did the reviewers assess the quality/validity of the included studies?

d) Were the essential data/studies adequately described?

\section{Data collection}

The main points that refer to IoT were extracted for each selected paper:

a) The journal and all the references

b) Paper type (conference, book etc)

c) Abstracts

d) Main body of each paper including the research questions and answers

e) Year of publication

When the data extraction was completed, all the selected journals were stored in a table with respect to the year of publication. The paper abstracts went under review in order to decide to continue the paper to the next phase or to reject it [6-8]; Figure 1.

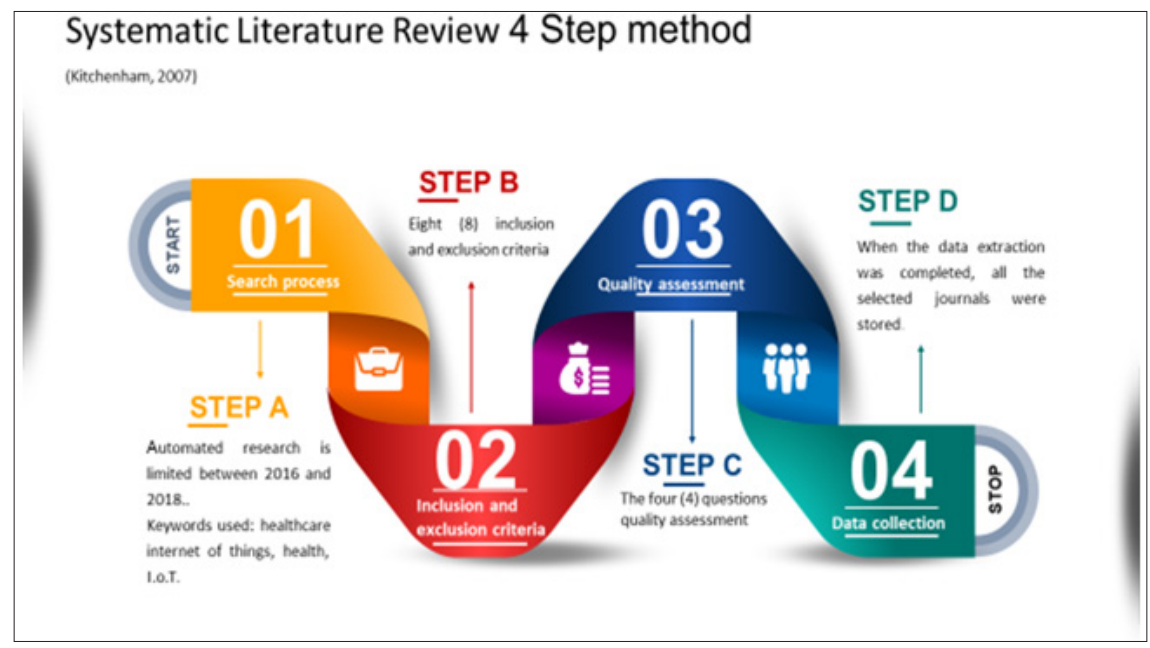

Figure 1: 1-4 steps method.

\section{Result}

The articles that were finally assessed and selected for this study are shown in Table 2. A thorough analysis of these papers was conducted. More specifically, in [9] Mustafa Abdullah Azzawi and his colleagues, regarding the review of literature for the IoT on health services, underline with particular interest, the wearing sensors worn to the body for providing information. This information reflects either biological features, biochemical characteristics or physiological characteristics of a human organism. This health monitoring framework has a specific architecture: the body sensing element network level, which has sensors applied to transportable 
devices and act as units for information acquisition. Such information are pressure, rate and temperature. The data collection from the sensors is transmitted to a tank where the user selects their processing. The next step includes nodes of further processing and analysis. Through the research of the above authors in other studies, the context of the IoT to health services is clearly recorded. Safety issues emerge as the primacy for the implementation of all domains of IoT in healthcare sectors. Similar issues seem to have been overcome by using an authentication mechanism. The proposed solution is evaluated to be reliable, mainly dependent on CoAP (Constrained Application Protocol) with ECC (Elliptic Curve Cryptography) algorithms. The proposed technique fits the IoT needs in restricted devices, whereas providing a strong coding sort.

Table 2: Selected articles.

\begin{tabular}{|c|c|c|c|}
\hline Year of Publication & Writers & Journal & Title \\
\hline 2017 & $\begin{array}{c}\text { Jun Qi , Po Yang, Geyong Min, Oliver Amft , Feng } \\
\text { Dong, Lida Xu }\end{array}$ & $\begin{array}{l}\text { Pervasive and Mobile } \\
\text { Computing }\end{array}$ & $\begin{array}{c}\text { Advanced internet of things for } \\
\text { personalized healthcare systems "A survey }\end{array}$ \\
\hline 2016 & Yue Hong , Yan Zeng , Xing Chen, Yuanjie Fan & $\begin{array}{l}\text { Journal of Industrial } \\
\text { Information Integration }\end{array}$ & $\begin{array}{c}\text { The internet of things in healthcare: An } \\
\text { overview }\end{array}$ \\
\hline 2016 & $\begin{array}{c}\text { Mustafa Abdullah Azzawi, Rosilah Hassan and } \\
\text { Khairul Azmi Abu Bakar }\end{array}$ & $\begin{array}{l}\text { International Journal of Applied } \\
\text { Engineering Research }\end{array}$ & $\begin{array}{l}\text { A Review on Internet of Things (IoT) in } \\
\text { Healthcare }\end{array}$ \\
\hline 2015 & $\begin{array}{l}\text { S. M. Riazul Islam, (Member, IEEE), Daehan } \\
\text { Kwak, MD. Humaun Kabiri, Mahmud Hossain, } \\
\text { Andkyung-Sup Kwak, (Member, IEEE) }\end{array}$ & IEEE Access & $\begin{array}{l}\text { The Internet of Things for Health Care: A } \\
\text { Comprehensive Survey }\end{array}$ \\
\hline
\end{tabular}

Another study, by Jun Qi et al. [10], makes a reference to the implementation of IoT to the provision of health services, presenting articles that record in which health cases the IoT implementation has specific impacts. In cases where a patient's health is in a critical condition, sensors connected to the patient give the appropriate information to the attending physician to take the necessary action. More specifically, older individuals living alone through ADL (Activity of Daily Living)automatic detection in IoT surroundings offer knowledge from articulation radiocarpal devices with integral sensors and extra sensors in their home space. The data assortment is based on activity detection. The projected framework has tried to classify differing types of activities, like short-run emergencies (e.g., fall, weakness) and long clinical assessment (e.g. toilet use, sleep) through a psychological feature approach, and has highlighted as a feature for providing continuous info. Additionally, the correlation of events were represented in time order and helpful conclusions is drawn.

Another way people can take advantage of the IoT technology is the remote management of the musculoskeletal system [9]. Patients with arthritis, a debilitating chronic illness, through self-management of an ICT (Information and communications technology) system, have the potential, through cost savings, to improve their clinical image. A customized, long-term, control system can provide information for patients like a health issue but not medication and a healing and control aid plan can be developed through medical advice. The latter aims to reduce transfers to hospitals and thereby reduce the overall cost of dealing with such diseases. Self-management of rehabilitation and function problems could be a customized method through an observation platform for stable health conditions like chronic heart issues, chronic pain and stroke victimization transportable scanning technologies. The aim of this new device is to assist patients maintain their state of health by setting life goals supported varied physical activity observation results providing a series of feedbacks to doctors. In order to provide information on the patient's physical activity accelerometers and auxiliary instruments, such as scales and pressure level monitors, also as environmental sensors (e.g. bed sensor, door detector, etc.) are used, observance patient's activities time sleep period, TV watching and food preparation.

A different approach that aims at helping the elderly individuals to achieve accurate measures in their homes and to hold out day by day activities without external assistance, is introduced in [11]. More specifically, it uses an easy approach on a way to use fashionable home appliances through the interaction with network file system consoles connected to the instrumentation with their sensible devices. The final users were the aged and therefore the caregivers. People who provide care to elderly people are health care staff, family or friends, whose responsibility is to record and upload educational videos and link NFC (Near Field Communication) tags through the application Privacy Impact Assessment (PIA). The end user then uses good devices (e.g., smartphone or tablet) with the appliance put into the touch the instrumentation with the NFC tag hooked up, and the video itself is mechanically compete back.

A very promising project has been also proposed in [12]. This program develops the same and objective resolution to permit early diagnosing of Alzheimers, higher watching of the effectiveness of treatment and improved cost-effectiveness of diagnostic protocols. The project develops a general library of call support computer code and a platform with totally different sorting strategies through a CDSS model (clinical decision support system) consisting of information acquisition and data storage stack, a knowledge process scale, and a presentation stage for user interaction. The precise purpose of this CDSS tool is that the planned DSI (Display Serial Interface) mode wherever it seems to possess been effectively tested. However, there are other projects that are either in progress or not yet published in scientific journals and could not be identified by this work by Jun Qi [10]. 
In 2016 a review paper was published by Yue Hong et. al [13], on the use and implementation of IoT in health by collecting work on similar topics. The most important issues emerged from this article were a product that introduces a kind of smart rehabilitation after an Internet-based health problem and was recently introduced to mitigate the problem of the minimum resources due to the increase in population aging. It is considered to develop a sensible town. The health care system, associate degree IoT-based, connects all offered resources as a network for health care activities like diagnosing, observation and remote surgical interventions via the web. The framework was dedicated to the extension of health care services from hospitals to homes. Wireless technology has been wide applied to intrinsic observation devices, the front of which is treated as a network administrator.

The system connects all offered care resources to patients (e.g. hospitals, rehabilitation centers, doctors, nurses, ambulances, auxiliary devices, etc.). The server includes a central information. The process help is chargeable for knowledge analysis, integration, important incident identification, and recovery ways. All networks measure connected to the web and supported by programs based on RFID(Radio Frequency Identification) technology. An automatic resource dispenser has additionally been developed to quickly determine recovery solutions that meet a collection of specific necessities from individual patients. One of the foremost basic objectives of those products is that the overall value reduction and therefore the effectiveness of doctors' actions. This guide consists of 3 parts: Master, Server, and Things. Master includes doctors, nurses and patients who have a special license for the system from end-user devices (e.g. smartphone, laptop or tablet). Sever acts as a central part of the whole health care system, responsible for making prescriptions, direction, information analysis, scheme construction and cognitive content management. Things relate to one hand any physical objects (including patients and human resources) and on the other hand related to WAN, (wide space network) multimedia technology, or the Short Message Service (SMS). In addition, regular devices can't be connected to the network, however the unit ordinarily utilized in current recovery conditions are also enclosed within the sensible rehabilitation system and are network-compatible. The effectiveness of the projected design has been verified in the past [14]. A smart device or healthcare system usually incorporates IoT detection technologies, which allow the healthcare system to monitor patients from a distance.

Islam [15] as an IEEE Member, published a review article on the IoT in health services. The IoT tending network (IoThNet) is one among the important components of IoT in health care. It supports access to the spine of IoT, facilitates the transmission and reception of medical knowledge, and permits the utilization of communications tailored to health care. Names and categories for the application of IoT to health are related in the IoThNet topology, architecture and platform. However, it should be noted that the proposed structures could be considered as a decent place to begin developing ideas within the IoT network on health. The topology of the IoThNet refers to the layout of various parts of Nursing. IoT attention network, indicates representative eventualities of uninterrupted health care atmosphere. It converts the heterogeneous computing and storage capabilities of static and mobile electronic devices like laptops, smartphones and medical device terminals into hybrid laptop networks. Medical devices with sensors connected to the patient's body can provide very important biological information. Knowledge is then recorded, analyzed and kept. The information from numerous sensors and machines are helpful and their analysis is doable for health specialists to watch patients from anyplace and also the knowledge to be a guide to any application of health protocols. The IoThNet design refers to defining and sorting of IoThNet's physical parts, practicality, organization, and principles for its operation techniques. The key to the present design has been known in ability problems with the IoT entryway and Wireless native space Network (WLAN)/WPAN, multimedia system streaming and secure communications between IoT Portals and Health Professionals.

\section{Conclusion and Recommendations}

This work raises a number of issues to be explored for future studies. This is also the technological assessment of the implementation of IoT in health, how to integrate into the strategic plan of the IoT health business and, finally, the management of technological change in health through the impact on society. Through this article, where health articles are synthesized on health, general health services are being developed and this may be a structural element for a range of solutions for people in need. In addition, it ought to be noted that the overall health services and protocols needed within the context of implementation could need minor modifications for his or her correct functioning and supported the situations needed for health care provision. Researchers around the world have started exploring numerous technological solutions to strengthen health care provision in an exceedingly approach that enhances existing services through the capabilities of the IoT. The present paper examines various aspects of IoT in health using the literature review methodology and presents various health network architectures and platforms that support the transmission and reception of data with Internet access in order to facilitate providers and recipients of medical services. This is done either through smartphones or other communication devices. In addition, the work provides elaborated analysis information regarding the capability of IoT to address medicine care, elder care, chronic unwellness watching, personal health, and health management with these new technologies. For further insight into market trends and making broad-based technologies a reference center, this paper offers a broad knowledge base for ongoing developments in online device connectivity and how medical gadgets and related health services have prompted the expansion of Internet-based healthcare services.

There has been a particularly scientific gap regarding the economic envelope of health services through IoT. Particular mention is made to the lack of research into the overall cost of such a technology service either in the public sector or in the private sector and at the same time a better understanding of IoT healthcare in data security and the impact on providers and recipients. Discussion on many necessary problems, like standardization, 
network kind, business models, quality and impact of services either at the economic or social level, as well as health information protection are expected to be the key idea for any analysis on health Internet-based care, even if the aforementioned services are publicly available. This paper also presents policies on digital public health and ethics in the interest of various stakeholders in the assessment of IoT healthcare technologies. In conclusion, the results of this research are expected to be useful for researchers either in health and IT sectors as well as financial analysts and policy makers in the health and public sector in general. It is therefore urgent in the near future to implement a technology in the health sector but also across the other sectors in general, with a proper assessment to develop advanced health care techniques and technologies and to be used by those involved to benefit of all.

\section{References}

1. Evans D (2013) The internet of things: How the next evolution of the internet is changing government. ECEG Everything. Proceedings of the European Conference one 44(8): 301-309.

2. Patill S, Pardeshi S (2018) Health monitoring system using IoT. International Research Journal of Engineering and Technology (IRJET)5(4): 1678-1682.

3. Song X, Wang X, Ma YY, Zhang D (2015) Extended AODV routing method based on distributed minimum transmission (DMT) for WSN. Intl J Electronics \& Comms 69(1): 371-381.

4. Höller J, Tsiatsis V, Mulligan C, Karnouskos S, Avesand S, et al. (2014) From machine-to-machine to the internet of things: Introduction to a new age of intelligence. Elsevier, Amsterdam, The Netherlands.

5. Singh B, Bhattacharya S, Chowdhary CL, Jat DS (2017) A review on internet of things and its applications in healthcare. Journal of Chemical and Pharmaceutical Sciences 10(1).
6. Kitchenham B (2007) Und Stuart Charters. EBSE 2007-001.Keele University and Durham University Joint Report, UK.

7. Kitchenham B, Charters S (2007) Guidelines for performing systematic literature reviews in software engineering. Technical Report EBSE 2007001, Keele University and Durham University Joint Report.

8. Kitchenham B, Brereton P, Budgen D, Turner M, Bailey J, et al. (2009) Systematic literature reviews in software engineering-A systematic literature review. Information and Software Technology 51(1): 7-15.

9. Rosilah M, Khairul (2016) A review on internet of things (IoT) in healthcare. International Journal of Applied Engineering Research 11: 10216-10221.

10. Qi J, Yang P, Xu L, Min G, Amft O, et al. (2017) Advanced internet of things for personalized healthcare system: A survey. Pervasive and Mobile Computing 41: 132-149.

11. Rafferty J, Nugent CD, Liu J, Chen L (2017) From activity recognition to intention recognition for assisted living within smart homes. IEEE Trans Hum Mach Sys 47(3): 368-379.

12. Antila K, Lötjönen J, Thurfjell L, Laine J, Massimini M, et al. (2013) The PredictAD project: Development of novel biomarkers and analysis software for early diagnosis of the Alzheimer's disease. Interface Focus 3(2): 20120072.

13. Yuehong Y, Zeng Y, Chen X, Fan Y (2016) The internet of things in healthcare: An overview. Journal of Industrial Information Integration 1: 3-13.

14. Islam SMR, Kwak D, Kabir H, Hossain M, Kwak KS (2015) The internet of things for health care: A comprehensive survey. IEEEACCESS 3: 678-708.

15. Sonawdekar S, Katkar G, Gaikwad M, Farhan M (2018) Smart Hospitals using IOT. International Journal of Scientific \& Engineering Research $9(3)$. 